Send your research letters to the editor, British Dental Journal, 64 Wimpole Street, LondonW1G8YSE-mail bdj@bda dentistry.org.uk

Priority will be given to letters less than 500 words long. Authors must sign the letter, which may be edited for reasons of space

\section{Advanced development}

Sir, a seven year old girl of Ghanaian origin presented to our department as a casualty patient complaining of pain from an exfoliating lower left D. On examination the lower left D was indeed exfoliating with the lower left four beginning to erupt. However, what was of particular interest about this child was the developmental stage of her dentition. Clinical examination revealed the lower left $\mathrm{D}$ to be the sole remaining deciduous tooth and that three second permanent molars were fully erupted. Radiological examination (see Figure 1) revealed closed apices of all four first permanent molars plus both upper central and lateral incisors. In addition all four third permanent molars were shown to be developing.

Furthermore how interesting it is that caries is present in both lower second permanent molars, the most recently erupted teeth. Although it is well reported that children of African origin are well advanced in dental development compared with Caucasian children, this noteworthy patient is five years ahead of our textbook averages! Incidentally a thirteen year old girl of Nigerian origin recently presented with fully erupted upper third permanent molars and pericoronitis of almost fully erupted lower third permanent molars. Although this second patient was just a teenager her dentition was most certainly adult.

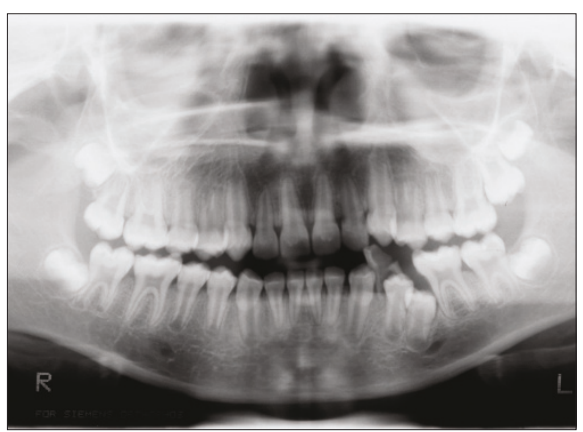

Figure 1

\section{Swinburne}

London

doi: $10.1038 /$ sj.bdj.4812337

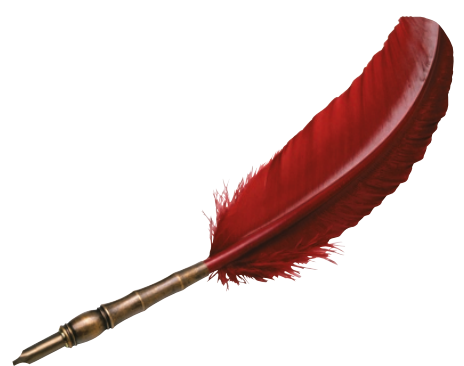

\section{Special precautions}

Sir, some of our colleagues may be unaware of the "adverse event" reports of osteonecrosis of the jaw of patients being treated with bisphosphonates. The majority of these reports refer to cancer patients following teeth extraction of other dental surgeries. This has led to warnings and special precautions for use being issued regarding the use of bisphosphonates.

It is suggested that a dental examination with appropriate preventive dentistry should be considered prior to treatment with bisphosphonates in patients with concomitant risk factors (e.g. cancer, chemotherapy, corticosteroids, poor oral hygiene).

While on treatment, these patients should avoid invasive dental procedures if possible. For patients who develop osteonecrosis of the jaw while on bisphosphonate therapy, dental surgery may exacerbate the condition. For patients requiring dental procedures, there are no data available to suggest whether discontinuation of bisphosphonate treatment reduces the risk of osteonecrosis of the jaw. Clinical judgment of the treating physician should guide the management plan of each patient based on individual benefit/risk assessment.

An example of this type of problem is illustrated by a lady born in 1924 who was diagnosed in 1999 with locally advanced breast cancer with widespread metastatic disease present in many bones (not involving the head). She was initially treated with tamoxifen and radiotherapy to the breast, maxilla and supraclavicular fossa. At the end of 2002 she was started on monthly injections of $4 \mathrm{mg}$ IV of Zometa. The patient had a pathological fracture of a femur in 2003 but otherwise remained very active.

The patient had regular dental inspections and was partially dentate with upper and lower partial dentures being worn. At a routine visit in June 2004 the patient complained of having a neuralgic type pain in the right maxilla area for the previous two months.
Examination showed upper 6/ to be present and mobile with there being a discharge of pus through the mucosa in the 5/ area. A radiograph was taken and a retained root was found in the 5/ area. Metronidazole was prescribed and a week later the 5/ root was surgically removed.

A month later the patient complained of pain associated with 6/ on biting and of a hole in the gum in the 5/ area. This prompted the practitioner to extract the $6 /$ and surgically examine the 5/ area in August. Five weeks later the patient presented in pain with a $2 \mathrm{~cm}$ by $10 \mathrm{~cm}$ area of uncovered bone in the molar and premolar regions of the right maxilla. The area was cleaned and closed using a buccal periosteal release flap.

At the end of November the patient was still having problems and was referred to the local maxillofacial unit regarding this apparent area of bone necrosis. An OPG $\mathrm{x}$-ray revealed an increase in height of the maxillary alveolar bone at the expense of half the maxillary antra bilaterally. Once again the necrotic bone was removed (the pathology being reported as dead membranous bone with no evidence of malignancy and no fungus being present). Sadly this patient recently died of carcinomatosis.

As many patients now regularly receive bisphosphonates (BNF 6.6.2) colleagues will need to be aware of these potential complications and liase with the drug prescriber.

\section{V. B. Nelson}

Cumbria

doi: $10.1038 /$ sj.bdj.4812338

\section{Improved stability}

Sir, it was a pleasure to read the useful article by Dr Gahan and Professor Walmsley concerning 'The neutral zone impression revisited' (BDJ 2005, 198:269). Without wishing to carp I note that the photograph in figure 6 shows that the wax up is significantly away from the plaster "lingual index". My feeling is that this technique is significantly improved if the neutral zone impression shaping is taken as being absolutely correct. 
This often results in the teeth being set to contact the labial aspect of the plaster index, but with all the space lingual to the teeth being filled with wax and subsequently acrylic. In this way the stability of the denture is much improved by ensuring there is contact of soft tissues all the way round the denture rather than just round the denture bases.

This is a most useful technique for making complete dentures more stable, especially in difficult denture cases. However I do feel that it is the filling of this whole area of the neutral zone shape, which contributes to its success. I do absolutely agree that the taking of the neutral zone at the correct occlusal vertical dimension is frequently the secret of success of this technique.

\section{M. Woodhead}

\section{Bristol}

doi: 10.1038/sj.bdj.4812338

\section{Journal clubs}

Sir, we read with interest the recent letter from Dr J. Ahearne regarding evidencebased dentistry, and the validity of clinical trials in general. ${ }^{1}$ As one of the goals of our departmental journal club is to teach critical appraisal skills, we decided to look at the two studies highlighted by Dr Ahearne, ${ }^{2,3}$ which had found opposite, and conflicting conclusions regarding the possible relationship between periodontal disease and adverse pregnancy outcome.

We used a 'debate-style' format ${ }^{4}$ for our meeting, and subsequently voted on the null hypothesis "there is no relationship between periodontal disease in pregnancy and adverse pregnancy outcome'. Firstly, two senior house officers presented one paper each, discussing the methodology, results and their own critical appraisal of the two papers. The debate was chaired by the Trust's Professor of Surgery (PAB), with 22 dental and doubly qualified members of the department in attendance.

The paper by Moore et $a l^{2}$ published in the $B D J$ appeared to be a wellconstructed large prospective cohort study, which took account of a number of identified confounding factors in the statistical analysis before concluding that there was no relationship between periodontal disease and pre-term birth or lower birth weight.

In contrast, the paper by Radnai et al published in the Journal of Clinical Periodontology, was a small-sample, retrospective case-control study, where the influence of cigarette smoking could not be controlled for by the statistical analysis due to the extent of difference in this factor between the cases and controls. It was pointed out during the debate that the choice of statistical test used to analyse the data may well have been incorrect in view of the non-normal distribution of the control group, so that the results as presented might well be misleading. In that respect, one of the authors of this letter (PA Brennan) who is on the editorial board of several clinical journals informed the debate that he would have rejected the manuscript for publication on that basis.

Perhaps unsurprisingly, the motion was carried unanimously in the vote.

We also considered an alternative motion: 'This house believes that there is no association between periodontal disease and adverse pregnancy outcome' (the distinction being that an association does not necessarily imply a causal relationship). Here the vote was more diverse, with several club members abstaining as 'don't knows'.

Dr Ahearne has spotted the geographic difference in patient population as a possible cause of differing study conclusions. We believe that by using a systematic process of critical appraisal, we have discovered that one of these studies is a far more reliable evidence source than the other. We advocate a similar type of regular journal club in the general practice setting, which not only contributes towards CPD, but also enables practitioners to develop critical appraisal skills. It is important not to fall into the trap of assuming that because a paper is published in a refereed journal, it must be sound. It is the responsibility of the reader to check the validity and applicability of research findings for themselves.

\section{H. Beckett \\ R. Ramsey \\ B. Thompson \\ P. A. Brennan \\ Portsmouth}

1. Ahearne J. Evidence based dentistry. Br Dent J 2004 197: 594

2. Moore $S$, Ide $M$, Coward $P Y$, Randhawa $M$, Borkowska E, Baylis R, Wilson R F. A prospective study to investigate the relationship between periodontal disease and adverse pregnancy outcome. Br Dent J, 2004; 197: 251-258

3. Radnai M, Gorzo I, Nagy E, Urban E, Novak T, Pal A. A possible association between preterm birth and early periodontitis. A pilot study. J Clin Periodontol 2004; 31: 736-741.

4. Gonzalez L. Referees make journal clubs fun. BMJ 2003; 326:106.

doi: $10.1038 /$ sj.bdj.4812338 